\title{
Genetik - Chance und Dilemma
}

\section{Anita Rauch}

Prof. Dr. med., Universität Zürich, Institut für Medizinische Genetik

\begin{abstract}
Genetik ist in aller Munde und nährt grosse Hoffnungen insbesondere auch im Rahmen der personalisierten Medizin. Demgegenüber stehen aber auch Sorgen um Diskriminierung und Missbrauch. Zum Beispiel hat gerade die Geburt zweier Mädchen in China, deren Erbgut im Rahmen einer künstlichen Befruchtung gentechnisch verändert wurde, für grossen Aufruhr gesorgt und grundlegende Debatten über ethische Aspekte der Gentherapie befeuert.
\end{abstract}

Die meisten jetzt praktizierenden Ärzte haben im Studium nur wenig Grundlagen der modernen Genetik mit auf den Weg bekommen. Angesichts des rasanten Fortschritts in der Genetik haben darum viele nur eine vage Vorstellung davon, was tatsächlich heute schon möglich ist und was uns in den nächsten Jahren erwartet. Dieser Artikel soll deshalb einen kurzen Überblick geben, wo wir heute in Sachen Medizinische Genetik stehen.

\section{Wichtige Erkenntnisse dank Fortschritt der Genomanalyse}

Dank des methodischen Fortschritts der Genomanalyse ist eine wichtige Erkenntnis der letzten beiden Jahrzehnte gereift: Ein beträchtlicher Anteil der chronischen Erkrankungen ist mehr oder weniger auf den angeborenen Defekt einzelner oder mehrerer Gene zurückzuführen. Dabei zeichnet sich in jüngster Zeit $a b$, dass "monogene» Krankheiten, bei denen schon der Defekt eines Gens krankheitsauslösend ist, gar nicht so selten sind wie lange Zeit vermutet. Internationale Studien deuten zum Beispiel darauf hin, dass jeder Zweihundertste in der Normalbevölkerung Anlageträger für Mutationen in den Genen BRCA1 oder 2 ist, welche das Risiko für Mamma-, Ovarial- oder Prostatakarzinom stark erhöhen (Maxwell et al., 2016). Eine noch unveröffentlichte Studie aus unserem Institut zeigt sogar, dass bei Einbeziehung von insgesamt 19 bekannten Genen für solch eine Krebsprädisposition ei-

Wir kennen heute mehrere Tausend monogene Krankheiten, die jedes Organsystem betreffen können.

ner von 50 in der Normalbevölkerung Anlageträger für ein hohes Risiko ist und dass des Weiteren einer von

\section{Résumé}

La génétique fait beaucoup parler d'elle et alimente de grands espoirs, notamment dans le cadre de la médecine personnalisée. Mais la discrimination qu'elle peut engendrer est également à l'origine de fortes préoccupations. La naissance de deux petites filles en Chine, dont le patrimoine génétique a été modifié lors d'une insémination artificielle, a ainsi suscité un vif émoi et des débats de fond sur les aspects éthiques de la thérapie génique.

20 ein etwas erhöhtes Risiko aufweist. Darüber hinaus kennen wir heute mehrere Tausend monogene Krankheiten, die jedes Organsystem betreffen können. Die meisten davon sind jedoch individuell selten und vielen Ärzten unbekannt, so dass sie vermutlich unterdiagnostiziert sind. Schätzungen gehen davon aus, dass 5-8\% der Bevölkerung an einer seltenen monogenen Krankheit leiden.

\section{Verbesserte Diagnose und Therapie für den Patienten}

Die neuen Hochdurchsatzverfahren für Genanalysen erlauben zunehmend eine effiziente Diagnostik und Beendigung der oft jahrelangen diagnostischen Odyssee der Betroffenen. Die KLV-Analysenliste als Pflichtleistungskatalog von Laboranalysen ermöglicht seit 2015 die Hochdurchsatzsequenzierung von bis zu mehreren hundert Genen als differentialdiagnostisches Werkzeug bei idiopathischen Symptomen. Dennoch werden heute noch nicht alle monogenen Krankheiten diagnostiziert. Ein Grund dafür ist, dass erst ca. ein Viertel unserer Gene einer bestimmten Krankheit zugeordnet werden konnten. Zudem ist eine grosse An- 


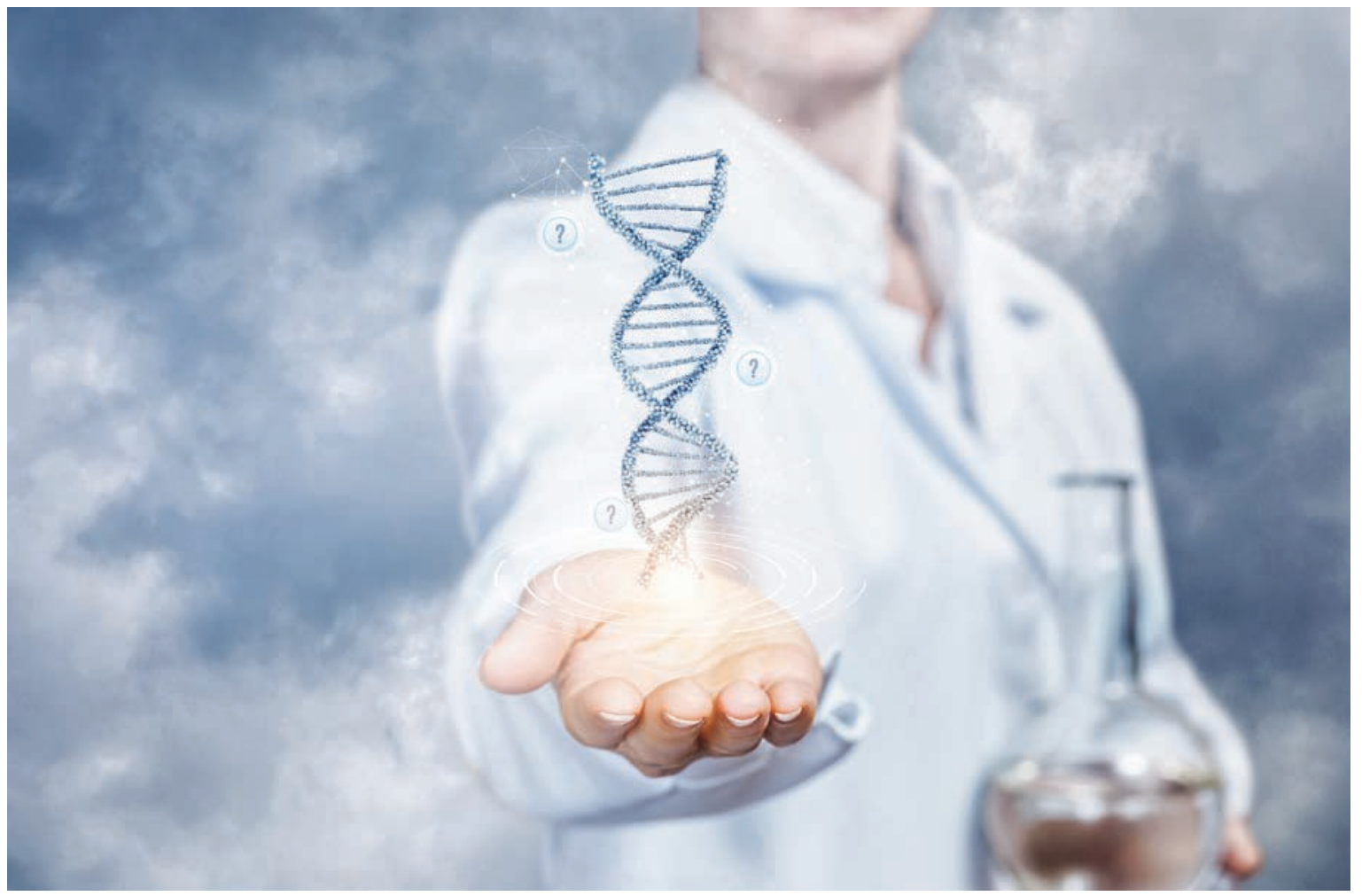

zahl von genetischen Abweichungen hinsichtlich ihrer Pathogenität noch unklar oder mit den aktuellen Analysemethoden noch nicht nachweisbar.

\section{Die neuen Hochdurchsatzverfahren für Gen- analysen erlauben zunehmend eine effiziente Diagnostik.}

Das Stellen einer ätiologischen Diagnose mittels Gentest erlaubt dabei nicht nur eine präzisere Prognose und bessere Krankheitsbewältigung, sondern auch eine pathophysiologisch begründete Behandlung und zunehmend sogar eine gezielte ursächliche Therapie. So stehen z.B. inzwischen zur Behandlung der im deutschsprachigen Raum relativ häufigen monogenen Cystischen Fibrose (CF) gezielte Medikamente zur Verfügung. Bei der CF kommt es aufgrund eines defekten Chloridkanals insbesondere zur chronischen Pankreasinsuffizienz und Lungenfibrose. Mutationen, welche zur Fehlregulierung des dabei defekten CFTR-Kanals führen, kann seit 2014 mit dem Wirkstoff Ivacaftor sehr effizient entgegengewirkt werden. Bei der häufigen Mutation p.F508del können seit 2018 mit dem Wirkstoff Tezacaftor die Anzahl funktioneller Chloridkanäle auf der Zelloberfläche vermehrt werden.

Eine weitere Erfolgsgeschichte ist die Behandlung der bei uns ebenfalls relativ häufigen, meist frühletalen spinalen Muskelatrophie. Diese kann durch Aktivierung des physiologisch rudimentären Zweitgens durch das 2017 zugelassene Medikament Nusinersen effizient aufgehalten werden. Noch effizienter zu sein verspricht die in Studien bereits erfolgreich bei der spinalen Muskelatrophie angewandte Genersatztherapie. Auch die PCSK9-Inhibitoren zur Behandlung familiärer Hypercholesterinämien zeigen eine durchschlagende therapeutische Wirkung. Diese für relativ seltene monogene Formen entwickelten Medikamente sind zudem selbst bei unspezifischer klinisch manifester Herzkrankheit infolge einer Arteriosklerose anwendbar und versprechen, die Behandlung breiter Patientenkreise zu beeinflussen.

\section{Neue Einsichten zu häufigen und komplexen Krankheiten}

Neben der Aufklärung monogener Krankheiten ermöglichte der technische Fortschritt in der Genetik in den letzten zehn Jahren auch zunehmend ein besseres Verständnis von häufigen und in der Regel auf komplexen Interaktionen zwischen Umwelt, Lifestyle und Genen beruhenden Krankheiten. Hierbei kommen immer grösser angelegte genomweite Assoziationsstudien («GWAS») zur Anwendung, welche eine Vielzahl von in der Bevölkerung häufigen genetischen Varianten zutage brachten. Diese tragen jeweils mit kleinem Effekt jedoch in der Summe signifikant zu zahlreichen häufigen Krankheiten bei. In den letzten fünf Jahren gibt es auch zunehmend Studien, die auf der Grund- 
lage häufiger Genvarianten Risikoaussagen für einzelne Krankheiten wie Arteriosklerose oder Schizophrenie treffen wollen («Polygenic Risk Scores»). Deren Validität für das einzelne Individuum ist jedoch sehr umstritten, so dass sie noch keinen Platz in der medizinischen Praxis gefunden haben. Dessen ungeachtet ermöglichte die Aufklärung genetischer Faktoren, die zu häufigen komplexen Krankheiten beitragen, schon vielfach einen tieferen Einblick in die Pathophysiologie dieser Krankheiten mit entsprechender Relevanz für die Entwicklung gezielterer therapeutischer Strategien. Dies war insbesondere bei Typ-2-Diabetes und Autoimmunkrankheiten der Fall (Übersicht bei Visscher et al., 2017).

\section{Fortschritt ermöglicht gezielte Prävention - und Designerbabys}

Die Diagnose eines angeborenen hohen Risikos für bestimmte Krebserkrankungen oder für Komplikationen zum Beispiel im kardiovaskulären Bereich ermöglicht eine individualisierte Früherkennung, chirurgische Prophylaxe und zunehmend auch medikamentöse Prävention zum Beispiel mittels Beta-Blockern oder Losartan beim Marfan-Syndrom. In manchen Populationen wird auch eine Primärprävention mittels Heterozygotenscreening für in der jeweiligen Population häufige Erbkrankheiten angeboten. So lassen sich z.B. Ashkenazy-Juden häufig bei Kinderwunsch oder sogar schon hinsichtlich der Partnerwahl auf Anlageträgerschaft für einige in dieser Population sehr häufige schwerwiegende frühkindliche Krankheiten untersuchen, welche bei den Kindern nur auftreten, wenn beide Eltern Anlageträger sind. Auch die gezielte vorgeburtliche Diagnostik familiärer Krankheiten wird durch den Nachweis familiärer Mutationen zunehmend möglich. Seit Ende 2017 ist in der Schweiz für Paare mit hohem Risiko, eine schwere Erbkrankheit an ihre Kinder zu vererben, auch eine Selektion diesbezüglich gesunder Embryonen im Rahmen der künstlichen Befruchtung erlaubt (Präimplantationsdiagnostik). Aufgrund der ethischen Bedenken hinsichtlich der Embryoselektion unterliegt die Präimplantationsdiagnostik in der Schweiz einer sehr strengen Regulierung. Die ethische Debatte hinsichtlich der Wegbereitung von Designerbabys wird seit der breiten Verfügbarkeit effizienter Genscheren mittels CRISPR-CAS9-Technologie seit 2012 aktueller denn je. Von chinesischen und koreanisch-amerikanischen Forschergruppen wurde schon gezeigt, dass die Korrektur eines Gendefekts bzw. das Einbringen einer genetischen Optimierung mittels Anwendung von CRISPR-CAS9 während einer künstlichen Befruchtung gelingen kann. Aufgrund un-

\section{Fit für die digitale Transformation?}

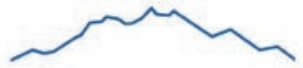

Trendtage Gesundheit

Luzern

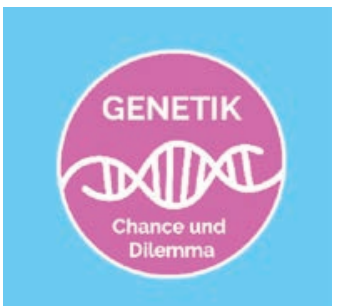

Am 27. und 28. März 2019 treffen sich an den Trendtagen Gesundheit im KKL Luzern einmal mehr über 600 Meinungs- und Entscheidungsträger des Gesund heitswesens. Der zweitägige Kongress befasst sich mit dem Thema "GENETIK - Chance und Dilemma».

Diagnose dank DNA-Analyse, zukunftsweisende Therapiemöglichkeiten dank Genom-Editierung, Prävention dank Epigenetik: Die Forschung am Erbgut eröffnet nie dagewesene Möglichkeiten, die die Medizin von morgen prägen. Dies stellt betroffene Akteure vor laufend neue Fragen, für die es dringend und laufend angepasste Lösungen braucht. Denn neues Wissen wird immer komplexer und schneller verfügbar. Neue Finanzierungsmodelle sind gefragt, ethische Fragen müssen geklärt werden. Politische und gesellschaftliche Aspekte erhalten eine neue Dimension. Wohin geht die Reise und welche Fragen müssen wir heute schon beantworten können?

Weitere Informationen und Anmeldung unter www.trendtagegesundheit.ch.

klarer Langzeitfolgen wurden solche editierten Embryonen jedoch bislang vernichtet (Ma et al., 2017). Im Herbst 2018 kam es diesbezüglich zum ethischen Dammbruch durch die Geburt zweier chinesischer Mädchen, die mittels CRISPR-CAS9 einen Resistenzfaktor gegen HIV-Infektionen erhalten haben sollen. Für den Moment lösten diese Experimente internationale Empörung aus. Weitere ethische Debatten ranken sich um die teilweise extrem hohen Kosten für die individualisierte bzw. gezielte (Gen-)Therapie.

Trotz der angesprochenen Diskussionen und Risiken geben die grossen Erfolge genetischer Anwendungen in der Medizin Grund zur Hoffnung auf eine erfolgreiche Weiterentwicklung massgeschneiderter Medizin bis hin zu einer personalisierten Gesundheitsvorsorge zum Wohle der Patienten.

\section{Bildnachweis}

(c) Nataliia Mysik | Dreamstime.com

\section{Literatur}

1 Maxwell KN, Domchek SM, Nathanson KL, Robson ME. Population Frequency of Germline BRCA1/2 Mutations. Journal of clinical oncology: official journal of the American Society of Clinical Oncology. Dec 2016;34(34):4183-5. 10.1200/jco.2016.67.0554.

2 Visscher PM, Wray NR, Zhang Q, Sklar P, McCarthy MI, Brown MA, Yang J. 10 Years of GWAS Discovery: Biology, Function, and Translation. Am J Hum Genet. 2017;101:5-22.

3 Ma H, Marti-Gutierrez N, Park SW, Wu J, Lee Y, Suzuki K, et al. Correction of a pathogenic gene mutation in human embryos. Nature. 2017:548(7668):413-9. doi: 10.1038/nature23305. 